\title{
Identifying and computing nonlinear normal modes
}

\author{
A. Cammarano ${ }^{1 *}$, A. Carrella ${ }^{1}$, L. Renson ${ }^{2}$, G.Kerschen ${ }^{2}$ \\ ${ }^{1}$ University of Bristol, Department of Aerospace Engineering, \\ Unversity Walk, BS8 1TR, Bristol,UK \\ ${ }^{2}$ University of Liege, Department of Aerospace and Mechanical Engineering, \\ 1, Chemin des Chevreuils, Liege, B-4000, BE
}

\begin{abstract}
Non linear normal modes offer a rigorous framework, both mathematical and physical, for theoretical and experimental dynamical analysis. Albeit still in its infancy, the concept of non linear normal modes has the potential of providing to both the academic and the industrial establishment a powerful tool for the analysis of non linear dynamical systems. However, in order to exploit the full potential of this theory (and its associated simulation capability), there is need to integrate it with other branches of non linear structural dynamics: namely, in order for the non linear normal modes of a real - physical - structure to be computed, there is need to identify and quantify its non linearity. In this paper, an identification method based on the measured Frequency Response Function (FRF) is employed to identify and quantify the system's non linearity before computing the system's non linear normal modes.
\end{abstract}

Introduction The last decades have witnessed a continuous demand for structures to become more light and efficient without loosing in safety and durability. This approach, which is well known in the aerospace engineering, is slowly influencing other fields of the automotive engineer as well as new branches of the civil engineer. In practice, the design process which relies on the theoretical and numerical modelling of a system and on the experimental observation for the identification and validation of these models, lacks methods which enables to account for the non linearities which occur in operational regime. In the last century the great advance in computational science and in numerical methods provided indispensable tools for solving the complex system of equations needed to model this type of structures where large displacements might not meet the hypothesis of linearity. This step is absolutely crucial for their analysis, but nevertheless not sufficient. The main problem is the definition of the model itself. How is it possible to associate a mathematical model with a given structure so that we are able to describe its dynamics? This science, known as identification, is the real question that we are not able to answer yet. The typology of non-linearity is generally an unknown of the problem as well as the parameters which characterize the equations. The identification of this information from experimental data is not easy and requires, in general some assumptions on the non-linearity [1]. Even in that case, it is not entirely clear which experiments are more useful for a full parameters' identification and what data are strictly necessary for the definition of a suitable mathematical model. This work aims to answer some of the questions still open in the world of non-linear structures. With this purpose in mind, the authors simulated numerically the behaviour of a non-linear systems with three degree of freedom. The data generated, i.e. the numerical FRFs are analysed with the method presented in [2], (also referred to as CONCERTO). The main advantage of this approach is that the structure is simulated numerically and both the type of non-linearity and the equation parameters are known. In this contest a combination of numerical and simulation procedures is used to enhance the identification and thus the prediction capability. After a short description of

*e-mail: andrea.cammarano@bristol.ac.uk 
the numerical method used to generate the data and the method behind CONCERTO, some results will be presented. In particular the test will show how this can be used for the identification of multi degree of freedom (MDOF) systems, highlighting its advantages and its limitations. Finally, a few considerations about the method will be presented and some possible directions for future works suggested.

\section{Continuation of forced, periodic response of non linear systems}

The forced response of discrete mechanical systems with $n$ degrees of freedom (DOFs) is considered, assuming that continuous systems (e.g., beams, shells or plates) have been spatially discretized using the finite element method. The equations of motion are

$$
\mathbf{M} \ddot{\mathbf{x}}(t)+\mathbf{C} \dot{\mathbf{x}}(t)+\mathbf{K} \mathbf{x}(t)+\mathbf{f}_{\mathrm{nl}}\{\mathbf{x}(t), \dot{\mathbf{x}}(t)\}=\mathbf{f}(t)
$$

where $\mathbf{M}$ is the mass matrix; $\mathbf{C}$ is the damping matrix; $\mathbf{K}$ is the stiffness matrix; $\mathbf{x}, \dot{\mathbf{x}}$ and $\ddot{\mathbf{x}}$ are the displacement, velocity and acceleration vectors, respectively; $\mathbf{f}_{\mathrm{nl}}$ is the non linear restoring force vector and $\mathbf{f}(t)$ is the external force vector.

The numerical method proposed here for the computation of the forced periodic response of non linear systems relies on the algorithm developed for the computation of non linear normal modes (NNMs), which are periodic responses of the undamped, unforced system [3]. The NNM algorithm relies on two main techniques, namely a shooting technique and the pseudo-arclength continuation method and is described below.

\subsection{Shooting Method}

The undamped, unforced equations of motion of system (1) can be recast into state space form

$$
\dot{\mathbf{z}}=\mathbf{g}(\mathbf{z})
$$

where $\mathbf{z}=\left[\begin{array}{ll}\mathbf{x}^{*} & \dot{\mathbf{x}}^{*}\end{array}\right]^{*}$ is the $2 n$-dimensional state vector, and star denotes the transpose operation, and

$$
\mathbf{g}(\mathbf{z})=\left(\begin{array}{c}
\dot{\mathbf{x}} \\
-\mathbf{M}^{-1}\left[\mathbf{K} \mathbf{x}+\mathbf{f}_{\mathrm{nl}}(\mathbf{x}, \dot{\mathbf{x}})\right]
\end{array}\right)
$$

is the vector field. The solution of this dynamical system for initial conditions $\mathbf{z}(0)=\mathbf{z}_{0}=$ $\left[\begin{array}{ll}\mathbf{x}_{0}^{*} & \dot{\mathbf{x}}_{0}^{*}\end{array}\right]^{*}$ is written as $\mathbf{z}(t)=\mathbf{z}\left(t, \mathbf{z}_{0}\right)$ in order to exhibit the dependence on the initial conditions, $\mathbf{z}\left(0, \mathbf{z}_{0}\right)=\mathbf{z}_{0}$. A solution $\mathbf{z}_{p}\left(t, \mathbf{z}_{p 0}\right)$ is a periodic solution of the autonomous system $(2)$ if $\mathbf{z}_{p}\left(t, \mathbf{z}_{p 0}\right)=$ $\mathbf{z}_{p}\left(t+T, \mathbf{z}_{p 0}\right)$, where $T$ is the minimal period.

The computation is carried out by finding the periodic solutions of the governing nonlinear equations of motion (2). In this context, the shooting method is probably the most popular numerical technique. It solves numerically the two-point boundary-value problem defined by the periodicity condition

$$
\mathbf{H}\left(\mathbf{z}_{p 0}, T\right) \equiv \mathbf{z}_{p}\left(T, \mathbf{z}_{p 0}\right)-\mathbf{z}_{p 0}=\mathbf{0}
$$

$\mathbf{H}\left(\mathbf{z}_{0}, T\right)=\mathbf{z}\left(T, \mathbf{z}_{0}\right)-\mathbf{z}_{0}$ is called the shooting function and represents the difference between the initial conditions and the system response at time $T$. For forced motion, the period $T$ of the response is known a priori.

The shooting method consists in finding, in an iterative way, the initial conditions $\mathbf{z}_{p 0}$ and the period $T$ that realize a periodic motion. To this end, the method relies on direct numerical time integration and on the Newton-Raphson algorithm.

Starting from some assumed initial conditions $\mathbf{z}_{p 0}^{(0)}$, the motion $\mathbf{z}_{p}^{(0)}\left(t, \mathbf{z}_{p 0}^{(0)}\right)$ at the assumed period $T^{(0)}$ can be obtained by numerical time integration methods (e.g., Runge-Kutta or Newmark schemes). In general, the initial guess $\left(\mathbf{z}_{p 0}^{(0)}, T^{(0)}\right)$ does not satisfy the periodicity condition (4). A Newton-Raphson iteration scheme is therefore to be used to correct an initial guess and to converge 
to the actual solution. The corrections $\Delta \mathbf{z}_{p 0}^{(k)}$ and $\Delta T^{(k)}$ at iteration $k$ are found by expanding the nonlinear function

$$
\mathbf{H}\left(\mathbf{z}_{p 0}^{(k)}+\Delta \mathbf{z}_{p 0}^{(k)}, T^{(k)}+\Delta T^{(k)}\right)=0
$$

in Taylor series and neglecting higher-order terms (H.O.T.).

The phase of the periodic solutions is not fixed. If $\mathbf{z}(t)$ is a solution of the autonomous system $(2)$, then $\mathbf{z}(t+\Delta t)$ is geometrically the same solution in state space for any $\Delta t$. Hence, an additional condition, termed the phase condition, has to be specified in order to remove the arbitrariness of the initial conditions. This is discussed in detail in [3]. For forced motion, the phase is fixed by the external forcing.

In summary, an isolated periodic solution is computed by solving the augmented two-point boundary-value problem defined by

$$
\mathbf{F}\left(\mathbf{z}_{p 0}, T\right) \equiv\left\{\begin{array}{c}
\mathbf{H}\left(\mathbf{z}_{p 0}, T\right)=0 \\
h\left(\mathbf{z}_{p 0}\right)=0
\end{array}\right.
$$

where $h\left(\mathbf{z}_{p 0}\right)=0$ is the phase condition.

\subsection{Continuation of Periodic Solutions}

Different methods for numerical continuation have been proposed in the literature. The so-called pseudo-arclength continuation method is used herein.

Starting from a known solution $\left(\mathbf{z}_{p 0,(j)}, T_{(j)}\right)$, the next periodic solution $\left(\mathbf{z}_{p 0,(j+1)}, T_{(j+1)}\right)$ on the branch is computed using a predictor step and a corrector step.

\section{Predictor step}

At step $j$, a prediction $\left(\tilde{\mathbf{z}}_{p 0,(j+1)}, \tilde{T}_{(j+1)}\right)$ of the next solution $\left(\mathbf{z}_{p 0,(j+1)}, T_{(j+1)}\right)$ is generated along the tangent vector to the branch at the current point $\mathbf{z}_{p 0,(j)}$

$$
\left[\begin{array}{c}
\tilde{\mathbf{z}}_{p 0,(j+1)} \\
\tilde{T}_{(j+1)}
\end{array}\right]=\left[\begin{array}{c}
\mathbf{z}_{p 0,(j)} \\
T_{(j)}
\end{array}\right]+s_{(j)}\left[\begin{array}{c}
\mathbf{p}_{z,(j)} \\
p_{T,(j)}
\end{array}\right]
$$

where $s_{(j)}$ is the predictor stepsize. The tangent vector $\mathbf{p}_{(j)}=\left[\mathbf{p}_{z,(j)}^{*} p_{T,(j)}\right]^{*}$ to the branch defined by (6) is solution of the system

$$
\left[\begin{array}{c}
\left.\frac{\partial \mathbf{H}}{\partial \mathbf{z}_{p 0}}\right|_{\left(\mathbf{z}_{p 0,(j)}, T_{(j)}\right)} \\
\left.\frac{\partial h}{\partial \mathbf{z}_{p 0}}\right|_{\left(\mathbf{z}_{p 0,(j)}\right)}
\end{array}\right]\left[\begin{array}{l}
\mathbf{p}_{z,(j)} \\
p_{T,(j)}
\end{array}\right]=\left[\begin{array}{l}
\mathbf{0} \\
0
\end{array}\right]
$$

with the condition $\left\|\mathbf{p}_{(j)}\right\|=1$. The star denotes the transpose operator. This normalization can be taken into account by fixing one component of the tangent vector and solving the resulting overdetermined system using the Moore-Penrose matrix inverse; the tangent vector is then normalized to 1 .

\section{Corrector step}

The prediction is corrected by a shooting procedure in order to solve (6) in which the variations of the initial conditions and the period are forced to be orthogonal to the predictor step. At iteration $k$, the corrections

$$
\begin{aligned}
\mathbf{z}_{p 0,(j+1)}^{(k+1)} & =\mathbf{z}_{p 0,(j+1)}^{(k)}+\Delta \mathbf{z}_{p 0,(j+1)}^{(k)} \\
T_{(j+1)}^{(k+1)} & =T_{(j+1)}^{(k)}+\Delta T_{(j+1)}^{(k)}
\end{aligned}
$$

are computed by solving the overdetermined linear system using the Moore-Penrose matrix inverse

$$
\left[\begin{array}{c}
\left.\frac{\partial \mathbf{H}}{\partial \mathbf{z}_{p 0}}\right|_{\left(\mathbf{z}_{p 0,(j+1)}^{(k)}, T_{(j+1)}^{(k)}\right)} \\
\left.\left.\frac{\partial h^{*}}{\partial \mathbf{z}_{p 0}}\right|_{\left(\mathbf{z}_{p 0,(j+1)}^{(k)}\right)}\right|_{\left(\mathbf{z}_{p 0,(j+1)}^{(k)}, T_{(j+1)}^{(k)}\right)} \\
\mathbf{p}_{z,(j)}^{*}
\end{array}\right]\left[\begin{array}{c}
\Delta \mathbf{z}_{p 0,(j+1)}^{(k)} \\
\Delta T_{(j+1)}^{(k)}
\end{array}\right]=\left[\begin{array}{c}
-\mathbf{H}\left(\mathbf{z}_{p 0,(j+1)}^{(k)}, T_{(j+1)}^{(k)}\right) \\
-h\left(\mathbf{z}_{p 0,(j+1)}^{(k)}\right) \\
0
\end{array}\right]
$$


where the prediction is used as initial guess, i.e, $\mathbf{z}_{p 0,(j+1)}^{(0)}=\tilde{\mathbf{z}}_{p 0,(j+1)}$ and $T_{(j+1)}^{(0)}=\tilde{T}_{(j+1)}$. The last equation in (10) corresponds to the orthogonality condition for the corrector step.

This iterative process is carried out until convergence is achieved. The convergence test is based on the relative error of the periodicity condition:

$$
\frac{\left\|\mathbf{H}\left(\mathbf{z}_{p 0}, T\right)\right\|}{\left\|\mathbf{z}_{p 0}\right\|}=\frac{\left\|\mathbf{z}_{p}\left(T, \mathbf{z}_{p 0}\right)-\mathbf{z}_{p 0}\right\|}{\left\|\mathbf{z}_{p 0}\right\|}<\epsilon
$$

where $\epsilon$ is the prescribed relative precision.

The system used for this work consists of three masses (namely $M_{1}, M_{2}$ and $M_{3}$ ) connected between each other and to the ground through springs and dashpots (as shown in Fig. 1). The dashpots and the spring characteristics are linear excepting for the spring connecting the first mass to the ground, which responds to a movement of $M_{1}$ with the elastic force $F_{e 1}$ given by

$$
F_{e 1}=K_{l} x_{1}+K_{n l} x_{1}^{3}
$$

where $x_{1}$ is the displacement of the first mass in comparison with its equilibrium position, $K_{l}$ is the linear stiffness of the spring and $K_{n l}$ is the coefficient of the non linear term. The forcing term $f(t)$ is a sinusoidal force with amplitude $F$ and is applied to $M_{1}$. The system, when the non linear term of $F_{e 1}$ is set to zero is linear and has three natural frequencies at $0.096 \mathrm{~Hz}, 0.226 \mathrm{~Hz}$ and $0.257 \mathrm{~Hz}$. The theory of non linear systems suggests that the position of the peaks in the frequency response is not influenced by the non-linear term and that for small forcing amplitude the frequency response tends to the linear response. In the case of non linear behaviour the amplitude of the frequency response function (FRF) has been evaluated considering the maximum amplitude of time response over a period. It's phase is computed using the Fourier Transform: the phase of the applied force is subtracting from the phase of the response. The identification tool uses this information to evaluate the characteristics of the system.

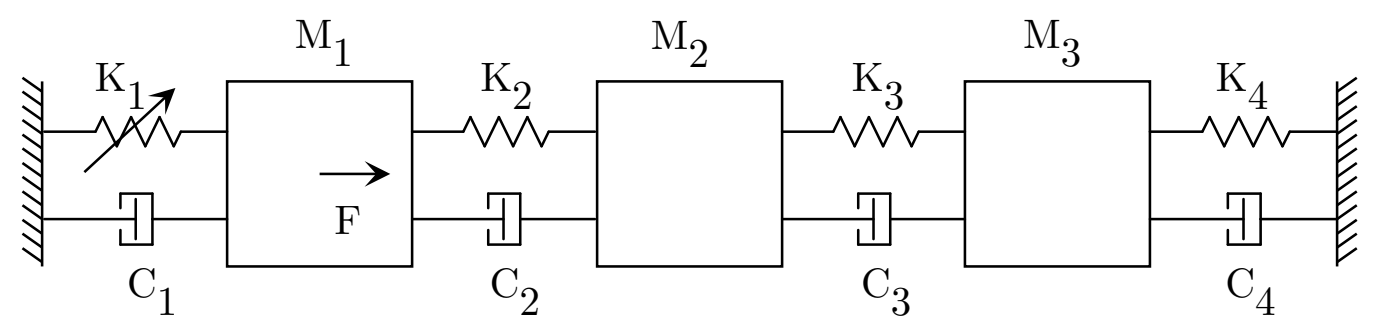

Figure 1: Schematic of the simulated system: the the arrow on the spring named $K_{1}$ indicates its non linear stiffness.

\section{Identification tool: theory}

The method used for the identification procedure is referred tp as CONCERTO and is described in [2], thus only a brief summary is given here. CONCERTO is based on the assumption that each peak dominates the response in correspondence of the resonant frequency and therefore the system can be thought as a single-degree-of-freedom (SDOF) system, with amplitude-dependent damping and/or stiffness - which are the most common classes of non linearity in engineering structures. The equation of motion for a SDOF is the same as equation 1 with the only difference that the coefficient of the equation are now scalar terms

$$
M \ddot{x}(t)+C \dot{x}(t)+K x(t)+f_{\mathrm{nl}}\{x(t), \dot{x}(t)\}=f(t)
$$

Assuming that the system responds at the same frequency as the excitation, the FRF is measured. The forcing term is a sinusoidal excitation with constant amplitude and variable frequency. For the point of the FRF at any given response amplitude, $x$, the functions $f_{\mathrm{nl}}(x)$ in equation 13 are in effect constants. This implies that it is possible to linearise the system at that specific response amplitude so that the systems FRF is given by 


$$
H(X, \omega)=\frac{1}{\omega_{o}^{2}(X)-\omega^{2}+j \omega_{o}^{2}(X) \eta(X)},
$$

where $\omega_{0}$ are the natural frequency and the modal loss factor at that given amplitude. It is important to note that the linearisation must refer to a given value of amplitude of displacement. The functions $\omega_{o}(X)$ and $\eta(X)$ can be extracted from the measured real and imaginary part of equation 14 as follows:

$$
\begin{gathered}
\omega_{o}(X)=\frac{\left(R_{2}-R_{1}\right)\left(R_{2} \omega_{2}^{2}-R_{1} \omega_{1}^{2}\right)+\left(I_{2}-I_{1}\right)\left(I_{2} \omega_{2}^{2}-I_{1} \omega_{1}^{2}\right)}{\left(R_{2}-R_{1}\right)^{2}+\left(I_{2}-I_{1}\right)^{2}}, \\
\eta(X)=\left|-\frac{\left(I_{2}-I_{1}\right)\left(R_{2} \omega_{2}^{2}-R_{1} \omega_{1}^{2}\right)+\left(R_{2}-R_{1}\right)\left(I_{2} \omega_{2}^{2}-I_{1} \omega_{1}^{2}\right)}{\omega_{o}\left[\left(R_{2}-R_{1}\right)^{2}+\left(I_{2}-I_{1}\right)^{2}\right]}\right|,
\end{gathered}
$$

where $R_{1}$ and $R_{2}$ are the real parts of the FRF at the amplitude $X$ and $I_{1}$ and $I_{2}$ its imaginary parts; $\omega_{1}$ and $\omega_{2}$ are the frequencies at which a certain amplitude of displacement occurs: by definition they these frequency values are always before and after the resonance frequency omega $a_{o}$. If the peak, due to non linear effect, is bent over itself so that a given value of displacement occurs more than two times, the program consider the extreme values of the interval [omega 1 , omega $a_{n}$ and the method loose in validity. This effect will be discussed in the following section and will be supported by graphical examples.

\section{Results and discussion}

In this section some results will be presented. CONCERTO has been used to identify the backbones of the FRF peaks. In the procedure the FRF relative to five different levels of excitation have been considered: $0.01 N, 0.03 N, 0.04 N 0.05 N$ and $0.1 N$. For each level of excitation, and each mass, the displacement values around one peak per time have been considered. The procedure has been repeated for each level of excitation and than for each peak. The results are presented in figure 2,3, and 4. For each mass the details of the peak and their backbones are presented. The backbones are evealuated both with the NNM algorithm described in section 1 (red dashed lines) and with FRF based method described in section 2 (black solid lines). In the first case the backbones have been evaluated considering the free oscillations of the undamped system. In the second case the natural frequency has been computed as function of the response amplitude according to equation 15. From each case, it can be noticed that the curves evaluated with the FRF method are generally very close to those computed with the simulation procedure. The error tends to be particularly small around the peak of the considered FRF and tend to diverge for smaller values of displacement. In previous works the FRF method has been applied only to weakly non linear systems where no unstable branch exists. In this work all the cases present both stable and unstable branches. An extension to this identification method to FRF with jumps (i.e. unstable branches) is presented in [4]. It is clear from this study (see for example figure $2(\mathrm{~d})$ ), that if the unstable branch of the FRF is provided the method works perfectly. On the other hand, the strong non linearity highlights a weakness of the method. In presence of strongly non linear effects, e.g. if the peak bends on itself (see for example figure 3(d) and $4(\mathrm{~d})$ ) there can be more than two frequencies at which a given amplitude is reached. In this case CONCERTO is not able to follow the peak because it looks for the minimum and maximum frequency at which the given displacement occurs. The results is that the found backbone heavily diverge from the real one as shown in the same figures. However, although of great academic interest, this occurrence is rare in practical engineering structures and therefore can be object of future studies.

Conclusion The combination of a continuation procedure with an identification method based on the FRF have shown to be a valid approach to study practical non linear structures. At this stage the data flow has been unidirectional from the simulation tools to the identification tool. The final goal of this study is the creation of a more complex tool able to extrapolate the parameter from experimental data and to iterate the passage between the identification program and the simulation tool to converge toward a model which can predict the behaviour of the analysed system. There are 

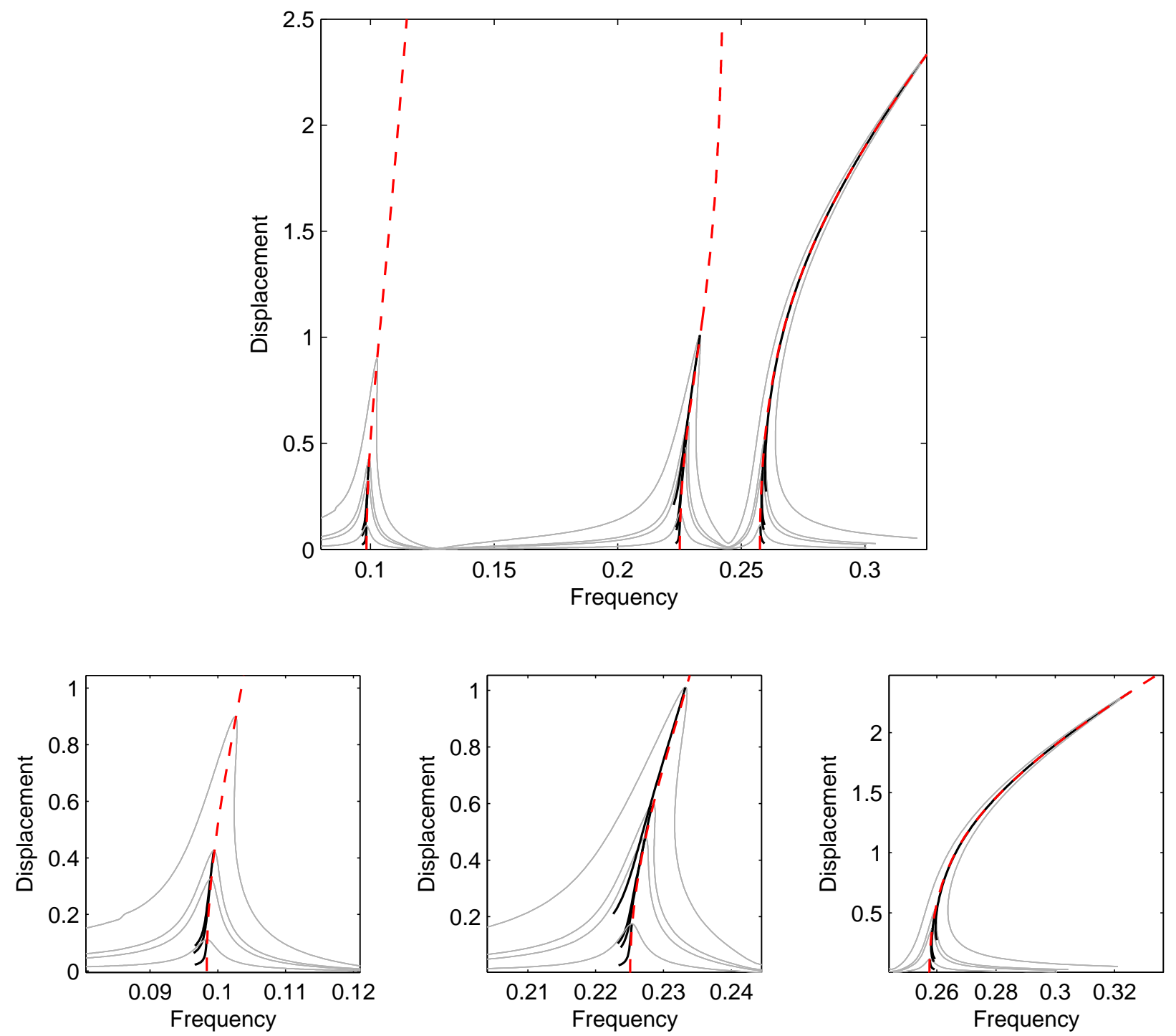

Figure 2: FRF of the displacement of the mass $M 1$ (gray solid lines) for different levels of eccitation and backbones line evaluated with the NNM code (red dashed lines) and with CONCERTO (black silid lines) 

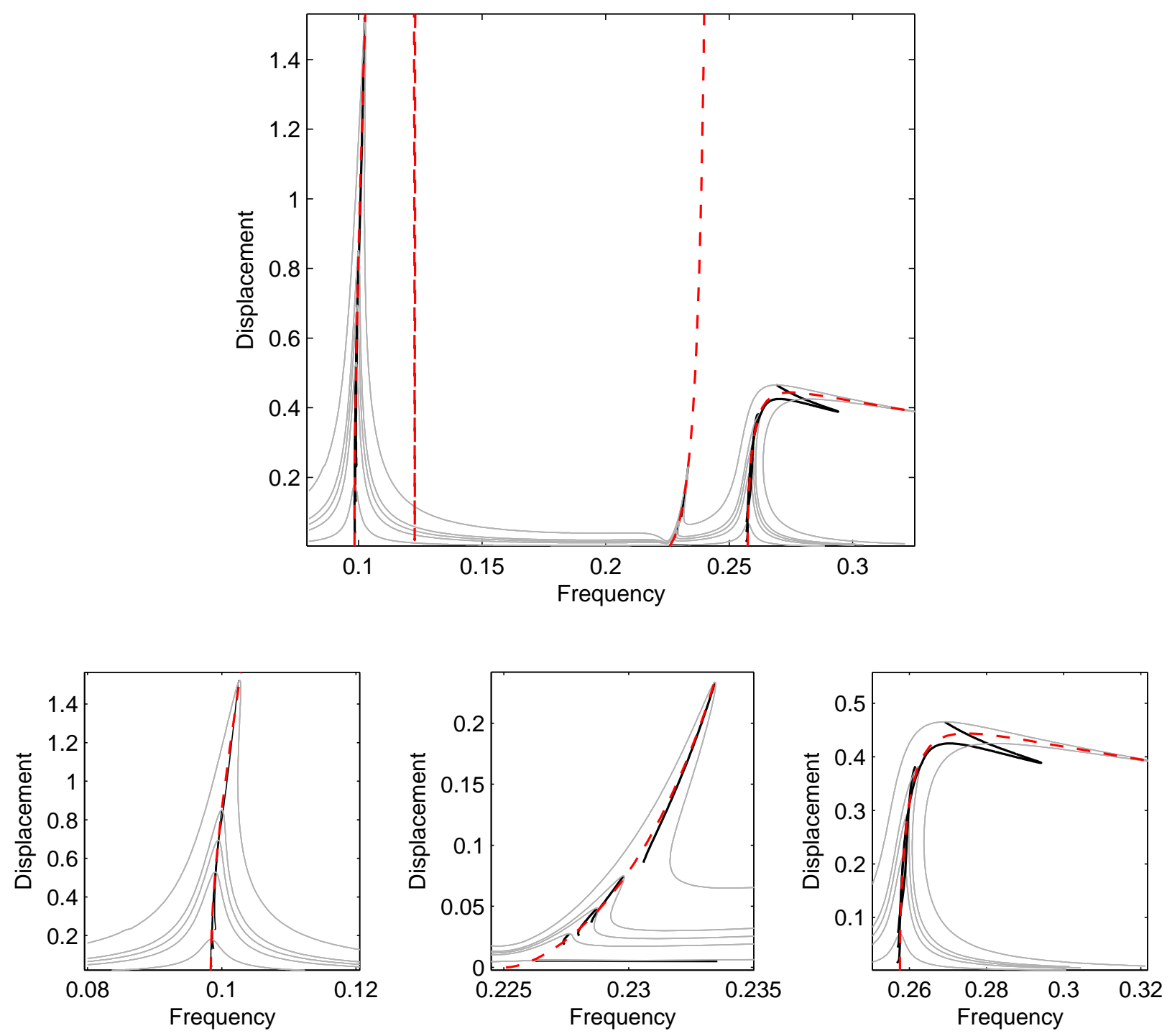

Figure 3: FRF of the displacement of the mass M2 (gray solid lines) for different levels of eccitation and backbones line evaluated with the NNM code (red dashed lines) and with CONCERTO (black silid lines) 

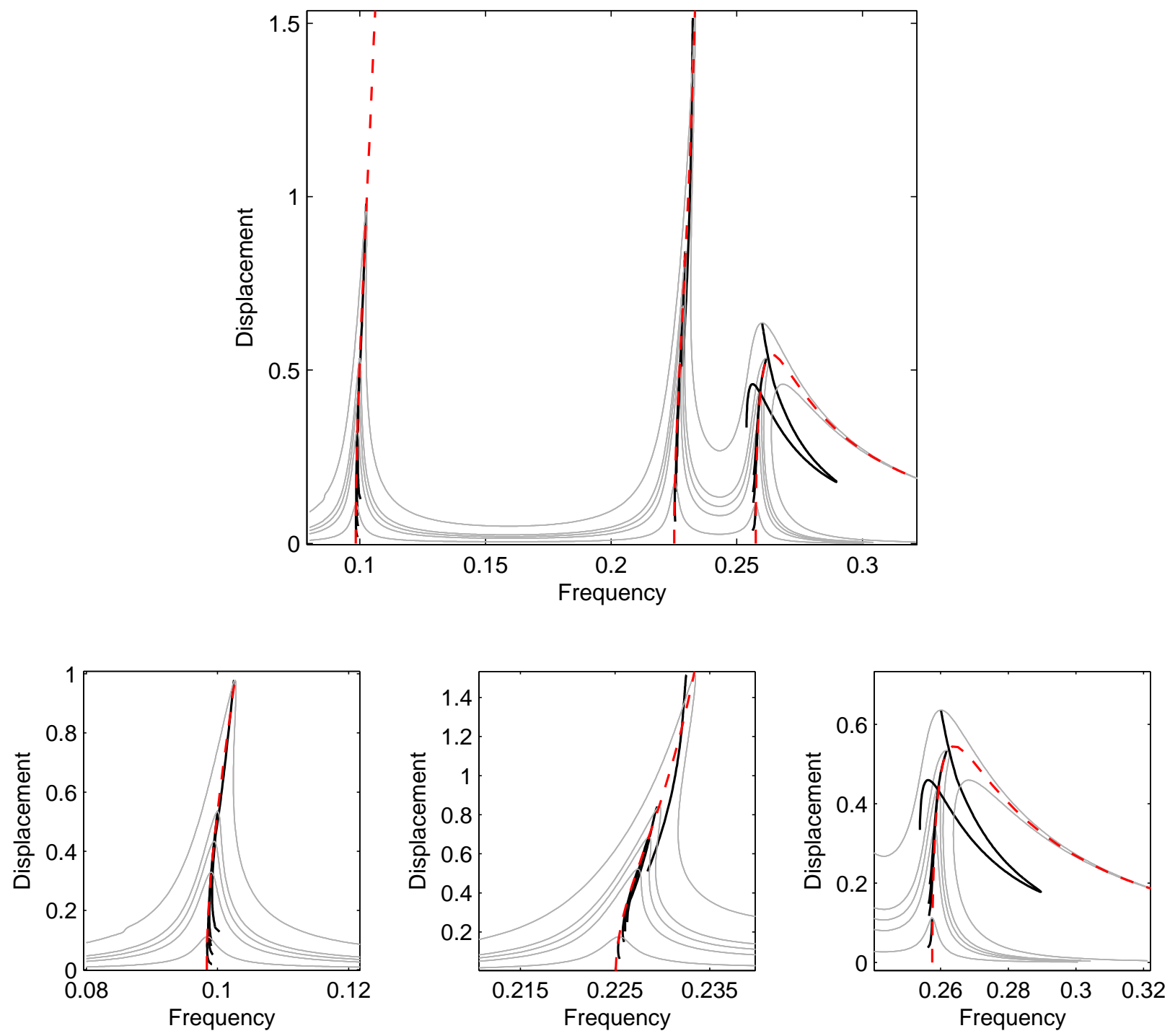

Figure 4: FRF of the displacement of the mass $M 3$ (gray solid lines) for different levels of eccitation and backbones line evaluated with the NNM code (red dashed lines) and with CONCERTO (black silid lines) 
several issues which have still to be addressed but the good matching between the NNM method and the FRF based procedure is definitely an interesting result for such an early stage. The next phase will be focussed also on understanding the interaction between the the modes to see if it is possible to reduce the error in the identification procedure. One issue to tackle is the definition of a suitable parameter domain for this study. Currently modal (natural frequency) and spacial (displacement) models are (mis)used. In fact, although it can be considered generally acceptable, the backbones found by the identification tools diverge if the displacement values are not close enough to the peak. Moreover there are some cases in which poorer results have been obtained (see figure 4(c)). Nevertheless, unless strong non linear behaviours occur, the trend of the backbone can be easily identified. The possibility to use weighted regression techniques will be strongly considered in future works to create a complete backbone from a limited set of data. This information is crucial for the estimation of the stiffness of the system.

\section{References}

[1] G. Kerschen, K. Worden, A.F. Vakakis, and J.C. Golinval. Past, present and future of nonlinear system identification in structural dynamics. Mechanical Systems and Signal Processing, 20(3):505-592, 2006.

[2] A. Carrella and DJ Ewins. Identifying and quantifying structural nonlinearities in engineering applications from measured frequency response functions. Mechanical Systems and Signal Processing, 2010.

[3] M. Peeters, R. Viguié, G. Sérandour, G. Kerschen, and J.C. Golinval. Nonlinear normal modes, part ii: Toward a practical computation using numerical continuation techniques. Mechanical systems and signal processing, 23(1):195-216, 2009.

[4] A. Carrella. Nonlinear identification using a frequency response functions with the jump. In IMAC XXX, Jacksonville, FL, volume 11, Feb 2012. 